\title{
Potencial de duas espécies de eucalipto na fitoestabilização de solo contaminado com zinco ${ }^{1}$
}

\author{
Potential of two species of eucalyptus in the phytostabilization of a soil contaminated \\ with zinc
}

\author{
Marcio Osvaldo Lima Magalhães ${ }^{2 *}$, Nelson Moura Brasil do Amaral Sobrinho ${ }^{3}$, Fabiana Soares dos Santos ${ }^{4}$ e \\ Nelson Mazur ${ }^{3}$
}

\begin{abstract}
Resumo - Com o objetivo de avaliar a técnica da fitoestabilização para remediar solo contaminado com zinco, foram utilizadas as espécies Eucalyptus urophylla e Eucalyptus saligna em conjunto com os resíduos siderúrgicos escória de aciaria e carepa de laminação. O experimento foi realizado em casa de vegetação na Universidade Federal Rural do Rio de Janeiro, utilizando-se um solo contaminado com Zn, coletado em área próxima ao pátio de minério do Porto de Itaguaí e ao local de disposição de resíduo perigoso da Cia Mercantil e Industrial Ingá em Itaguaí-RJ. O substrato foi tratado com dois agentes inertizantes: um resíduo industrial com característica alcalina (escória de aciaria) nas doses de 4 e $6 \%$ e outro com alto teor de óxido de ferro usado como adsorvente (carepa de laminação), em dose única de 1\%. Após o transplantio das mudas, foram feitas coletas do substrato para determinação do $\mathrm{pH}$ e o fracionamento de $\mathrm{Zn}$ nas diferentes formas químicas. Verificou-se que o substrato, não tratado, apresentava alto teor de zinco nas frações fitodisponíveis. Os tratamentos causaram a redução nas concentrações de $\mathrm{Zn}$ nas formas químicas hidrossolúveis e trocáveis e aumento nas fases mais estáveis. A menor dose de escória de aciaria foi suficiente para provocar diminuição nas concentrações de zinco em solução, sendo esse efeito evidenciado pelo desenvolvimento das plantas, enquanto a maior dose de escória de aciaria promoveu maior crescimento das espécies. A espécie que obteve melhor desenvolvimento foi o E. urophylla, entretanto, a que apresentou maior acúmulo total de Zn foi o E. saligna.
\end{abstract}

Palavras-chave - Eucalipto. Solos-teor de zinco. Fitorremediação.

\begin{abstract}
With the aim of assessing the phytoremediation process to improve the soil contamination with zinc, it was used Eucalyptus urophylla and Eucalyptus saligna with slag from steelmaking and scales from hot-strip mills. The experiment was conducted in a greenhouse at the Universidade Federal Rural do Rio de Janeiro, using a soil contaminated with zinc, collected in an area near the ore yard at Itaguaí harbor as well at the local of disposition of hazardous waste of Mercantil and Industrial Ingá Co. at Itaguaí-RJ. The substrate was treated with two inertizing agents: industrial waste with alkaline caracteristics (steelmaking slag) in 4 and $6 \%$ doses and another one with high content of iron oxide used as adsorption material (hot-strip mill scale) at single dose of $1 \%$. After seedling transplant, the substrate was collected in order to determine $\mathrm{pH}$ and zinc fractionation in its different chemicals forms. It was verified that the not treated substrate showed high zinc content in bioavaiable fractions. This caused a reduction in the Zinc concentration in the water-soluble and exchangable forms and increase in more stable phases. The smallest dose of steelmaging slag was sufficient to cause a reduction of zinc concentrations solutions and this effect was showed clearly in the plant development, while the highest dose of steelmaging slag promote higher development in the species. The specie that obtained better development was E. urophylla, that one showing higher zinc content was E. saligna.
\end{abstract}

Key words - Eucalipto. Soils-zinc content. Phytoremediation.

\footnotetext{
*Autor para correspondência

${ }^{1}$ Recebido para publicação em 05/05/2010; aprovado em 04/04/2011

Parte da Dissertação de Mestrado do primeiro autor

${ }^{2}$ Departamento de Solos, Universidade Federal Rural do Rio de Janeiro, UFRural-RJ, Brasil, marciomagalhaes@gmail.com

${ }_{3}^{3}$ Departamento de Solos, Universidade Federal Rural do Rio de Janeiro, UFRural-RJ, BR 465, km 7, 23.851-970, Seropédica-RJ, Brasil

${ }^{4}$ Universidade Federal Fluminense, Av. dos Trabalhadores, 420,Vila Santa Cecília, 27.255-125, Volta, Rio de Janeiro, Brasil
} 


\section{Introdução}

A recuperação de áreas impactadas está entre as prioridades da sociedade, tendo assim uma grande demanda comercial e ambiental por técnicas que possam minimizar ou recuperar essas áreas (JULIATTI et al., 2002).

Atualmente, há considerável interesse no desenvolvimento de estratégias que sejam eficientes e duráveis na remediação de solos contaminados com metais pesados (CONCAS et al., 2004). As condições ambientais e a biodisponibilidade dos metais pesados são fatores importantes para a escolha do método de remediação a ser utilizado (MULLIGAN et al., 2001).

$\mathrm{Na}$ técnica de imobilização ou contenção química, há redução da solubilidade e toxicidade dos metais pesados, entretanto sem reduzir o teor total no solo (BASTA; MCGOWEN, 2004). Várias técnicas são descritas para realizar a imobilização química, entre elas, utilização de matéria orgânica (CASTALDI et al., 2000; CASTALDI; MELIS, 2004), carbonatos e óxidos de cálcio (DEROME, 2000; LOMBI et al., 2002), além de resíduos industriais com características alcalinas, como escórias de siderurgia (CHEN et al., 2000) e resíduos ricos em óxidos de ferro (PEREIRA, 2009).

Diversos trabalhos demonstram a eficiência da fitorremediação, sendo considerada como promissora técnica de reabilitação de áreas contaminadas (CASTALDI et al., 2005; GARBISU, 2002; KAMNEV, 2003;). As árvores, por produzirem maior biomassa e acumularem maior quantidade de metais, nas raízes e no caule e contribuírem no controle da erosão são de grande interesse em programas de fitoestabilização de sítios contaminados com metais pesados (BRUNNER et al., 2008; GRAZZIOTTI et al., 2003;). Devido às características de crescimento rápido, sistema radicular bastante desenvolvido e facilidade de adaptação a condições estressantes (DELL; DAPING, 1995), o eucalipto apresenta um grande potencial para emprego em programas de fitoestabilização (ACCIOLY, 2004).

Diante do exposto, este trabalho teve como objetivo avaliar a técnica da fitoestabilização para remediar solo contaminado com zinco, utilizando as espécies Eucalyptus urophylla e Eucalyptus saligna em conjunto com os resíduos siderúrgicos escória de aciaria e carepa de laminação.

\section{Material e métodos}

O experimento foi realizado em casa de vegetação localizada no campus da Universidade Federal Rural do Rio de Janeiro (UFRRJ), no município de Seropédica-RJ. A instalação das unidades experimentais seguiu o delineamento inteiramente casualizado composto por 3 tratamentos e 4 repetições, com cultivo de 2 espécies de eucalipto.
Foram preparadas 24 unidades experimentais em vasos com capacidade de $5 \mathrm{~L}$ com o posterior transplantio das mudas de Eucalyptus urophylla e Eucalyptus saligna.

As unidades experimentais utilizadas foram: $\mathrm{T} 1$ Substrato (testemunha), T $2-$ Substrato $+4 \%$ EA $+1 \%$ CL e T 3 - Substrato + 6\% EA + 1\% CL, onde, E. A (Escória de Aciaria) e CL (Carepa de Laminação).

O substrato utilizado foi proveniente de escavações do solo do pátio de minério, localizado no porto de Itaguaí, na Baía de Sepetiba, Itaguaí-RJ. $\mathrm{O}$ material removido foi disposto em área próxima ao aterro de resíduos gerados pela Cia Mercantil e Industrial Ingá, classificado como resíduo perigoso. As amostras do substrato foram coletadas segundo a NBR 10.007 (ABNT, 2004). Após coleta, as amostras foram secas ao ar, destorroadas, peneiradas e homogeneizadas. Para determinação das concentrações pseudototais de $\mathrm{Zn}$ (TAB. 1) as amostras foram trituradas em almofariz de ágata e, posteriormente, realizada a extração com água régia (ISO 11466, 1995). O valor de $\mathrm{pH}(1: 2,5)$ e as concentrações de $\mathrm{Ca}, \mathrm{Mg}, \mathrm{K}, \mathrm{P}, \mathrm{Na}$ e $\mathrm{Al}$ foram determinadas pelo método proposto pela Embrapa (1997) e apresentados na Tabela 1.

Para realizar a imobilização química, foram utilizados como inertizantes dois resíduos gerados pela Companhia Siderúrgica Nacional-CSN: um com característica alcalina, escória de aciaria $(41,87 \%$ de $\mathrm{CaO}$; 4,63\% de $\mathrm{MgO} ; 35,54 \%$ de $\mathrm{SiO} ; 172,3 \mathrm{mg} \mathrm{kg}^{-1}$ de $\mathrm{Zn}$ ), e outro, como adsorvente, carepa de laminação, com alto teor de ferro (76,44\% de Fe; 196,6 $\left.\mathrm{mg} \mathrm{kg}^{-1} \mathrm{de} \mathrm{Zn}\right)$.

Posteriormente, para se definir a proporção adequada do resíduo inertizante alcalino (escória de aciaria), foi realizado um ensaio de curva de neutralização, que consistiu em adicionar quantidades crescentes de escória de aciaria, ao solo, na proporção de: $1 \% ; 2 \% ; 3 \% ; 4 \% ; 5 \%$ e $6 \%$ do resíduo alcalino. Esta mistura permaneceu incubada a $70 \%$ da capacidade de retenção do substrato por 7 dias até a estabilização do $\mathrm{pH}$. Verificou-se que para atingir os valores de $\mathrm{pH} 6$ e 7 era necessário adicionar as doses de 4 e $6 \%$ de Escória de Aciaria, respectivamente. Para avaliar o potencial do resíduo carepa de laminação na adsorção de $\mathrm{Zn}$, foi construída uma isoterma de adsorção, utilizando-se o modelo de Langmuir, e determinado o valor de adsorção máxima para $\mathrm{Zn}$ que foi de $454,5 \mathrm{mg} \mathrm{kg}^{-1}$. Em função desse valor definiu-se a dose de $1 \%$ para o resíduo carepa de laminação.

O solo contaminado foi seco ao ar, destorroado, peneirado em tamis de malha de 4,5 mm e homogeneizado. Os resíduos escória de aciaria e carepa de laminação foram misturados ao substrato, manualmente, dentro de sacos plásticos para que ocorresse sua homogeneização. 
Tabela 1 - Características químicas e teor de argila da amostra do substrato utilizado no experimento

\begin{tabular}{ccccccccccccccc}
\hline $\mathrm{Na}$ & $\mathrm{Ca}$ & $\mathrm{Mg}$ & $\mathrm{K}$ & $\mathrm{H}+\mathrm{Al}$ & $\mathrm{Al}$ & $\mathrm{S}$ & $\mathrm{T}$ & $\mathrm{V}$ & $\mathrm{m}^{1}$ & $\mathrm{Zn}$ & $\mathrm{pH}$ & $\mathrm{Corg}$. & $\mathrm{P}$ & Arg. \\
\hline$-------------------\mathrm{cmol}_{\mathrm{c}} \mathrm{dm}^{-3}------------------$ & & $\%$ & & $\mathrm{mg} \mathrm{kg}^{-1}$ & & $\mathrm{~g} \mathrm{~kg}^{-1}$ & $\mathrm{~g} \mathrm{dm}^{-3}$ & $\mathrm{~g} \mathrm{~kg}^{-1}$ \\
\hline 7,8 & 15,8 & 6,2 & 0,4 & 7,4 & 2,6 & 30,2 & 37,6 & 80 & 7,93 & 5761 & 4,4 & 23,9 & 26 & 310 \\
\hline
\end{tabular}

${ }^{1}$ Saturação por $\mathrm{Al}$

Em seguida foram incubados a $70 \%$ da capacidade de retenção do substrato durante um período de 30 dias. As mudas de Eucalyptus urophylla e Eucalyptus saligna estavam com idade aproximada de 120 dias e foram obtidas no Instituto de Floresta da Universidade Federal Rural do Rio de Janeiro.

O método utilizado para o fracionamento geoquímico do $\mathrm{Zn}$ foi o utilizado no trabalho de Santos et. al., (2007) que utiliza as seguintes soluções extratoras: água (fração hidrossolúvel: F1); cloreto de magnésio $1 \mathrm{~mol} \mathrm{~L}^{-1}$ (fração trocável; F2); DTPA (fração óxidos amorfos; F3), ácido acético $0,043 \mathrm{~mol} \mathrm{~L}^{-1}$ (fração carbonato; $\mathrm{F} 4$ ), e água régia (ISO , 1995) (fração F5; Zn ocluso e associado a outras formas recalcitrantes).

Durante o período experimental foi realizada a avaliação da altura das plantas, utilizando-se régua milimetrada, sendo a primeira avaliação realizada 15 dias após o transplantio, com avaliações subseqüentes em intervalos de 15 dias. Para o ajuste das equações de crescimento foi utilizado o método da regressão linear. No final do experimento as plantas foram coletadas, separadas em raiz, caule e folhas, e, posteriormente, lavadas em água destilada e deionizada. Foram deixadas para secagem em temperatura ambiente por um dia e, posteriormente, secas em estufa a $70{ }^{\circ} \mathrm{C}$ até atingirem peso constante.

O material para digestão foi obtido após moagem tanto das folhas, caule e das raízes, em moinho tipo Willey com malha de $2 \mathrm{~mm}$. As concentrações de $\mathrm{Zn}$ foram determinadas a partir da digestão nitroperclórica, na proporção de 6:1, pelo método de Tedesco et al., (1995). Com base nas concentrações e produção de matéria seca, foram calculadas as quantidades acumuladas dos metais pesados nas raízes, caule e folhas. Nos extratos obtidos de solo e planta foram determinadas as concentrações de $\mathrm{Zn}$, por espectrofotometria de absorção atômica, empregando-se o equipamento VARIAN - AA600 com LQ $0,20 \mathrm{mg} \mathrm{kg}^{-1} \mathrm{LD}$ de 0,7 para $\mathrm{Zn}$.

Foram efetuadas as seguintes análises estatísticas: Teste de Lilliefors para verificação da normalidade, Teste de Cochran e Bartlett para verificar a homogeneidade das variâncias, análise de variância, teste de médias Tukey ao nível de 5\% de significância.

\section{Resultados e discussão}

A concentração pseudototal de zinco (TAB. 1) no substrato esteve acima do valor de intervenção, considerando o cenário mais restritivo de Área de Proteção Máxima/Agrícola (CETESB, 2005), apresentando concentrações cerca de 13 vezes acima desse valor, evidenciando assim que o substrato estava contaminado $\mathrm{e}$ indicando a necessidade de intervenção.

A redução significativa no teor de $\mathrm{Zn}$ nas frações químicas de maior biodisponibilidade (frações F1 eF2) foram verificadas com a aplicação dos resíduos inertizantes (FIG. 1), apresentando valores de apenas $5 \%$ do total no tratamento com a menor dose de escória aciaria (4\%). $\mathrm{Na}$ maior dose, não foi detectado $\mathrm{Zn}$ na fração $\mathrm{F} 1$. O decréscimo nas frações F1 e F2 foram acompanhados do aumento nas fases mais estáveis (F3, F4 e F5). Esses resultados demonstram que os inertizantes utilizados foram eficientes na redução da biodisponibilidade do $\mathrm{Zn}$.

A redução de Znnas formas químicas biodisponíveis e o conseqüente aumento nas fases mais estáveis, nos tratamentos que receberam os resíduos inertizantes, quando comparados à testemunha, demonstraram que a elevação do pH pela aplicação da Escória de Aciaria propiciou a precipitação do Zn, na forma de carbonatos e potencializou a adsorção específica na superfície de óxidos de Fe, Mn e Al do solo e dos óxidos de Fe da Carepa de Laminação, decorrente do aumento de carga líquida negativa na superfície dos óxidos (GUPTA; SINHA, 2007).

A redução de Znnas formas químicas biodisponíveis e o conseqüente aumento nas fases mais estáveis, nos tratamentos que receberam os resíduos inertizantes, quando comparados à testemunha, demonstraram que a elevação do $\mathrm{pH}$ pela aplicação da escória de aciaria propiciou a precipitação do $\mathrm{Zn}$, na forma de carbonatos e potencializou a adsorção específica na superfície de óxidos de $\mathrm{Fe}, \mathrm{Mn}$ e Al do solo e dos óxidos de Fe da carepa de laminação, decorrente do aumento de carga líquida negativa na superfície dos óxidos (GUPTA; SINHA, 2007).

A Figura 2 apresenta a altura do Eucalyptus urophylla e E. saligna em função dos dias após o transplantio (DAP) nos diferentes tratamentos. Observa-se que ambas as espécies desenvolvidas no substrato sem adição de escória de 


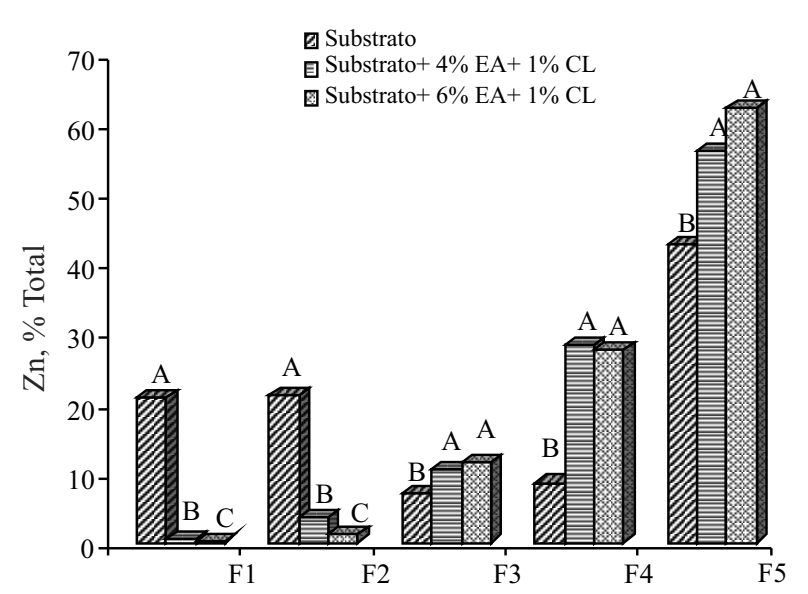

Figura 1 - Percentagem do total de Zn nas frações F1, F2, F3, F4 e F5 em função dos diferentes tratamentos. Valores seguidos pela mesma letra não diferem significativamente pelo Teste de Tukey, ao nível de 5\% de significância. EA, Escória de Aciaria; CL, Carepa de Laminação. F1: extração com água; F2: extraído com $\mathrm{MgCl}_{2}$; F3: extraído com DTPA; F4: extraído com ácido acético-; F5: extraído com água régia

aciaria e carepa de laminação (tratamento 1) não resistiram e morreram aos 30 dias após a implantação do experimento. Nesse tratamento, as plantas apresentaram, antes de morrerem, sintomas como: curvatura vertical das folhas, morte das gemas apicais e perda das folhas, sintomas também encontrados por Soares et al. (2005) provocados por toxicidade à Zn. Segundo Gichner et al. (2006), alto teor de Zn no solo, inibiu fortemente o crescimento de plantas, em comparação com plantas crescidas em solos não-poluídos, podendo apresentar diferentes respostas ao estresse, manifestados por sintomas visíveis, como a inibição do crescimento, danos ou clorose (PAVLÍKOVÁ et al., 2008). Provavelmente, o baixo $\mathrm{pH}$ observado neste tratamento (TAB. 1), favoreceu a maior biodisponibilidade do $\mathrm{Zn}$ (FIG. 1), sendo esses níveis possivelmente fitotóxicos para essas duas espécies de eucalipto.

Ao final do experimento verificou-se que a altura do E. urophylla variou de aproximadamente $113 \mathrm{~cm}$ (tratamento 2) a $137 \mathrm{~cm}$ (tratamento 3). Enquanto para a E. saligna a diferença na altura foi menor, atingindo $75 \mathrm{~cm}$ na dose de $4 \%$ de escória e $79 \mathrm{~cm}$ na maior dose (FIG. 2). Esses resultados demonstram que a espécie $E$. urophylla apresentou melhor resposta aos tratamentos do que o E. saligna. Esse maior desenvolvimento implica em maior produção de biomassa, sendo de grande interesse em programas de fitoestabilização de áreas contaminadas com metais pesados (GRAZZIOTTI et al., 2003).

Aproduçãodematériaseca(TAB.2)foiinfluenciada pela adição dos resíduos alcalino e adsorvente. Verifica se que há uma diferenciação entre a maior e a menor dose de escória de aciaria, sendo essa diferença significativa para todos os parâmetros avaliados, com exceção do ramo, para ambas as espécies. No tratamento 3 a espécie $E$. urophylla teve um aumento na massa seca, em relação ao tratamento 2, de aproximadamente $21 \%$ para folha, $40 \%$ para o caule, $59 \%$ para a raiz e $34 \%$ na biomassa total. Entretanto, para a espécie E. saligna esse aumento, em relação ao tratamento 2 , foi de $26 \%$ nas folhas, $36 \%$ no caule, $26 \%$ na raiz e $27 \%$ para a biomassa total. Dessa forma, o ganho em massa seca total na maior dose de escória de aciaria, em relação à menor, foi superior para o
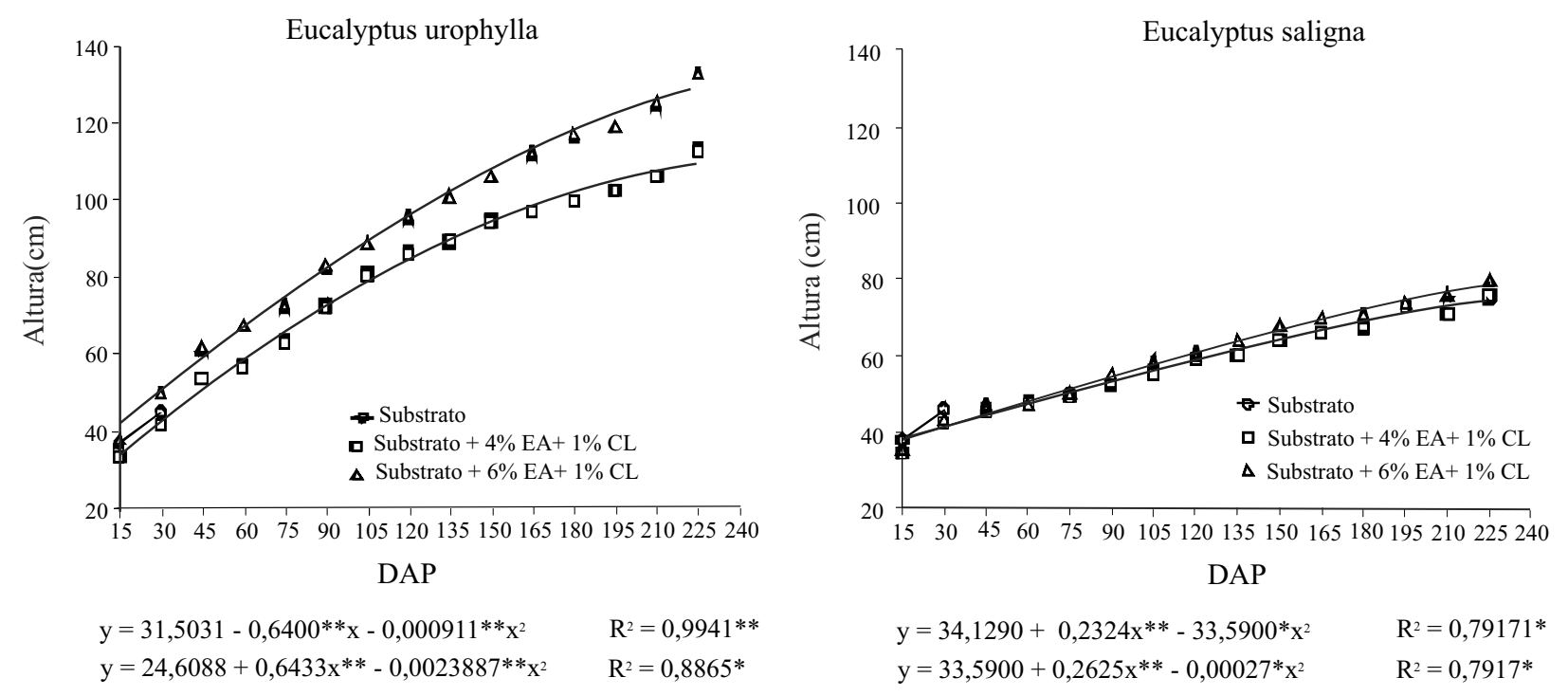

Figura 2 - Altura, em cm, das espécies Eucalyptus urophylla e Eucalyptus saligna cultivadas em substrato contaminado zinco, submetido a dois tratamentos. EA, Escória de Aciaria; CL, Carepa de Laminação; DAP, dias após o plantio 
E. urophylla, cerca de 34\%, em comparação ao E. saligna, com $27 \%$. Essa diferenciação entre os tratamentos foi provocada, possivelmente, pela maior biodisponibilidade dos metais no tratamento 2, em relação ao tratamento 3 (FIG. 1). Comparando a produção de massa seca na parte aérea entre as espécies, observa-se que E. urophylla teve maior produção do que E. saligna (TAB. 2).

Quanto à massa e o volume radicular, observase que tanto para E. urophylla quanto para E. saligna há diferença significativa entre os tratamentos 2 e 3 . No tratamento 3 , houve um aumento de $59 \%$ e $26 \%$ para o E. urophylla e E. saligna, respectivamente, quando comparado ao tratamento 1 . O volume de raiz produzido no tratamento 2 foi o mesmo para ambas as espécies. Já no tratamento 3, diferentemente da parte aérea, E. saligna apresentou um volume maior $\left(89 \mathrm{~cm}^{3}\right)$ do que E. urophylla $\left(73 \mathrm{~cm}^{3}\right)$, indicando que houve maior exploração do substrato pelas raízes.

Tabela 2 - Matéria seca (g) das folhas, caules, ramos e raízes e volume de raiz $\left(\mathrm{cm}^{3}\right)$ das espécies de eucalipto, cultivadas em substrato contaminado com $\mathrm{Zn}$

\begin{tabular}{|c|c|c|c|c|c|}
\hline & Espécie & $\mathrm{T} 1 *$ & $\mathrm{~T} 2 * *$ & $\mathrm{~T} 3 * * *$ & C.V. \\
\hline \multirow{2}{*}{ Folhas } & E. urophylla & $0,9 \mathrm{C}$ & $28,1 \mathrm{~B}$ & $34,1 \mathrm{~A}$ & \multirow{2}{*}{4,1} \\
\hline & E. saligna & $0,3 \mathrm{C}$ & $23,7 \mathrm{~B}$ & $30,0 \mathrm{~A}$ & \\
\hline \multirow{2}{*}{ Caules } & E. urophylla & $0,7 \mathrm{aC}$ & $18,1 \mathrm{aB}$ & $25,4 \mathrm{aA}$ & \multirow{2}{*}{3,2} \\
\hline & E. saligna & $0,6 \mathrm{aC}$ & $12,7 \mathrm{bB}$ & $16,7 \mathrm{bA}$ & \\
\hline \multirow{2}{*}{ Ramos } & E. urophylla & \pm & $4,8 \mathrm{bA}$ & $6,2 \mathrm{bA}$ & \multirow{2}{*}{5,8} \\
\hline & E. saligna & \pm & $7,7 \mathrm{aA}$ & $8,9 \mathrm{aA}$ & \\
\hline \multirow{2}{*}{ Raízes } & E. urophylla & $0,5 \mathrm{C}$ & $16,0 \mathrm{~B}$ & $25,6 \mathrm{~A}$ & \multirow{2}{*}{11,9} \\
\hline & E. saligna & $0,3 \mathrm{C}$ & $15,9 \mathrm{~B}$ & $20,2 \mathrm{~A}$ & \\
\hline \multirow{2}{*}{ Total } & E. urophylla & $2,1 \mathrm{aC}$ & $67,0 \mathrm{aB}$ & $90,2 \mathrm{aC}$ & \multirow{2}{*}{6,8} \\
\hline & E. saligna & $1,2 \mathrm{bC}$ & $59,6 \mathrm{bB}$ & $75,8 \mathrm{bA}$ & \\
\hline \multirow{2}{*}{ Vol. Raiz } & E. urophylla & $*$ & $57,0 \mathrm{~B}$ & $73,0 \mathrm{~A}$ & \multirow{2}{*}{2,4} \\
\hline & E. saligna & $*$ & $57,0 \mathrm{~B}$ & $89,0 \mathrm{~A}$ & \\
\hline
\end{tabular}

\pm Plantas que morreram aos 30 dias após o plantio. Médias seguidas pela mesma letra não diferem significativamente pelo teste de Tukey a $5 \%$. As letras maiúsculas na horizontal comparam os tratamentos e letras minúsculas na vertical comparam as espécies, para cada tratamento e parte vegetal. C.V. - coeficiente de variação; *T1 - Substrato; **T2 - Substrato + 4\% EA + 1\% CL; ***T3 - Substrato + 6\% EA + 1\% CL. EA, escória de aciaria; CL, carepa de laminação

Tabela 3 - Teores de $\mathrm{Zn}\left(\mathrm{mg} \mathrm{kg}^{-1}\right)$ na raízes, folhas, ramos e caules, por plantas de E. urophylla e $E$ saligna em função dos tratamentos

\begin{tabular}{|c|c|c|c|c|c|}
\hline \multicolumn{6}{|c|}{ Teores $\left(\mathrm{mg} \mathrm{kg}^{-1}\right)$} \\
\hline & Espécie & $\mathrm{T} 1 *$ & $\mathrm{~T} 2 * *$ & $\mathrm{~T} 3 * * *$ & C.V. \\
\hline \multirow{2}{*}{ Folhas } & E. urophylla & $4590,7 \mathrm{aA}$ & $78 \mathrm{bB}$ & $45,2 \mathrm{aC}$ & \multirow{2}{*}{5,1} \\
\hline & E. saligna & $1829,8 \mathrm{bA}$ & $100,5 \mathrm{aB}$ & $49,9 \mathrm{aC}$ & \\
\hline \multirow{2}{*}{ Caules } & E. urophylla & $2749,4 \mathrm{bA}$ & $63,9 \mathrm{aB}$ & $41,7 \mathrm{aB}$ & \multirow{2}{*}{5,8} \\
\hline & E. saligna & $5336,7 \mathrm{aA}$ & $50,3 \mathrm{aB}$ & $36,7 \mathrm{bB}$ & \\
\hline \multirow{2}{*}{ Ramos } & E. urophylla & \pm & $68,8 \mathrm{bA}$ & $49,8 \mathrm{aB}$ & \multirow{2}{*}{5,2} \\
\hline & E. saligna & \pm & $100,5 \mathrm{aA}$ & $49,9 \mathrm{aB}$ & \\
\hline \multirow{2}{*}{ Raízes } & E. urophylla & $6137,3 \mathrm{bA}$ & $676,2 \mathrm{bB}$ & $412,5 \mathrm{bC}$ & \multirow{2}{*}{3,1} \\
\hline & E. saligna & $9433,2 \mathrm{aA}$ & $890,5 \mathrm{aB}$ & $903,3 \mathrm{aB}$ & \\
\hline
\end{tabular}

Plantas que morreram aos 30 dias após o plantio. Médias seguidas pela mesma letra não diferem significativamente pelo teste de Tukey a 5\%. As letras maiúsculas na horizontal comparam os tratamentos e letras minúsculas na vertical comparam as espécies, para cada tratamento e parte vegetal. n.s. - não significativo; C.V. - coeficiente de variação, *T1 - Substrato; **T2 - Substrato + 4\% EA + 1\% CL; ***T3 - Substrato + 6\% EA + 1\% CL. EA, escória de aciaria; CL, carepa de laminação 
A produção de raízes é uma característica importante para a fitoestabilização de áreas contaminadas com metais pesados, pois protege o solo da erosão, reduz a lixiviação, favorece a agregação e a atividade microbiana do solo (CARNEIRO et al., 2002).

As concentrações de $\mathrm{Zn}$ na parte aérea e nas raízes foram influenciadas pela adição dos resíduos no substrato contaminado (TAB. 3). No tratamento 1, sem adição de resíduos, E. urophylla apresentou em suas folhas teores de $\mathrm{Zn}$ de $4.590,7 \mathrm{mg} \mathrm{kg}^{-1}$, cerca de 6 vezes acima dos valores considerados como níveis críticos de toxicidade $\left(697,8 \mathrm{mg} \mathrm{kg}^{-1}\right)$ encontrados por Soares et al., (2005), para essa espécie, e muito superior à faixa de 100-400 mg kg-1, considerada fitotóxica para o crescimento de outras espécies (KABATA-PENDIAS; PENDIAS, 2001).

Houve redução acentuada nas concentrações de $\mathrm{Zn}$ nas folhas nos tratamentos 2 e 3 , sendo essa redução de 59 e 102 vezes menor, respectivamente, do que as concentrações observadas no tratamento 1 , além de estar abaixo do nível crítico de toxicidade para a espécie $\left(697,8 \mathrm{mg} \mathrm{kg}^{-1}\right.$ de $\left.\mathrm{Zn}\right)$. Para as folhas essa redução entre os tratamentos foi significativa, sendo, no tratamento 3 , cerca de $42 \%$ menor do que no tratamento 2 . Pode-se também verificar que no tratamento 2, o teor de $\mathrm{Zn}$ nas folhas foi de $78 \mathrm{mg} \mathrm{kg}^{-1}$, valor acima da faixa considerada adequada (40-60 $\left.\mathrm{mg} \mathrm{kg}^{-1}\right)$ para Eucalipto proposto por Dell e Daping (1995).

Para E. saligna, no tratamento 1, o teor de Zn nas folhas também é elevado, chegando a ser cerca de 3 vezes o valor considerado tóxico para o eucalipto (SOARES et al, 2005). No tratamento 2, ocorreu uma redução, observando-se o teor de $100,5 \mathrm{mg} \mathrm{kg}^{-1}$, valor abaixo do nível crítico de toxicidade. No tratamento 3, o teor de $\mathrm{Zn}$ nas folhas foi de 49,9 $\mathrm{mg} \mathrm{kg}^{-1}$ e apesar de ocorrer essa redução, esse valor apresenta se dentro da faixa considerada adequada para o eucalipto (DELL; DAPING, 1995).

No caule das plantas de E. urophylla houve uma redução nas concentrações de $\mathrm{Zn}$ de 43 vezes no tratamento 2 , em relação ao tratamento 1 e não houve diferença significativa entre os tratamentos 2 e 3 . Para $E$. saligna a redução foi de aproximadamente 100 vezes no tratamento 2 e também não houve diferença significativa entre os tratamentos 2 e 3.

As concentrações de $\mathrm{Zn}$ nas raízes diminuíram com a aplicação dos resíduos alcalino e adsorvente, ocorrendo uma redução para $E$. urophylla cerca de 9 vezes para o tratamento 2 e de 15 vezes no tratamento 3, quando comparados com o tratamento 1. Para E. saligna essa redução foi de 11 vezes para o tratamento 2 e não houve diferença significativa entre os tratamentos 2 e 3 .

Os teores de $\mathrm{Zn}$ nas raízes de E. saligna são significativamente superiores ao de E. Urophylla; todavia, ao compararmos as concentrações na parte aérea, ocorre o inverso.

A capacidade da planta imobilizar o metal nas raízes, limitando sua translocação para a parte aérea, é um dos mecanismos de tolerância de algumas espécies de plantas aos metais pesados (GARBISU; ALKORTA, 2001).

Esses resultados explicam a diferença significativa entre os tratamentos 2 e 3 no desenvolvimento e acúmulo de massa seca das plantas. O crescimento da planta pode

Tabela 4 - Acúmulo de Zn nas folhas, caule, raiz e total, por plantas de E. urophylla e E. saligna em função dos tratamentos

\begin{tabular}{|c|c|c|c|c|c|}
\hline \multicolumn{6}{|c|}{ Acúmulo $\left(\mu \mathrm{g}_{\text {planta }}{ }^{-1}\right)$} \\
\hline & Espécie & $\mathrm{T} 1 *$ & $\mathrm{~T} 2 * *$ & $\mathrm{~T} 3 * *$ & C.V. \\
\hline \multirow{2}{*}{ Folhas } & E. urophylla & 4131,6 bA $(45,3)$ & $2191,8 \mathrm{aB}(15,1)$ & $1541,3 \mathrm{aB}(11,4)$ & \multirow{2}{*}{12,6} \\
\hline & E. saligna & $548,9 \mathrm{aC}(8,2)$ & 2381,1 aA $(14,4)$ & $1497,0 \mathrm{aB}(7,1)$ & \\
\hline \multirow{2}{*}{ Caules } & E. urophylla & 1924,6 bA $(21,1)$ & $1156,6 \mathrm{aB}(8,0)$ & $1056,2 \mathrm{aB}(7,9)$ & \multirow{2}{*}{18,1} \\
\hline & E. saligna & 3308,7 aA $(49,5)$ & 617,3 bB $(3,7)$ & 613,5 bB $(3,0)$ & \\
\hline \multirow{2}{*}{ Ramos } & E. urophylla & \pm & 330,9 bA $(2,3)$ & 309,3 bA $(2,3)$ & \multirow{2}{*}{21,9} \\
\hline & E. saligna & \pm & 793,8 aA $(4,7)$ & $448,7 \mathrm{aB}(2,6)$ & \\
\hline \multirow{2}{*}{ Raízes } & E. urophylla & $3068,7 \mathrm{aB}(33,6)$ & 10839,5 aA $(74,7)$ & $10551,8 \mathrm{bA}(78,4)$ & \multirow{2}{*}{10,8} \\
\hline & E. saligna & $2830,0 \mathrm{aC}(42,3)$ & $12711,6 \mathrm{aB}(77,1)$ & $18202,1 \mathrm{aA}(87,2)$ & \\
\hline \multirow{2}{*}{ Total } & E. urophylla & $9125 \mathrm{aB}(100)$ & 14519 aA (100) & 13462 bA (100) & \multirow{2}{*}{8,6} \\
\hline & E. saligna & 6688 bB (100) & $16483 \mathrm{aA}(100)$ & $20757 \mathrm{aA}(100)$ & \\
\hline
\end{tabular}

\pm Plantas que morreram aos 30 dias após o plantio. Valores entre parênteses representam a percentagem em relação ao total; Médias seguidas pela mesma letra não diferem significativamente pelo teste de Tukey a 5\%; As letras maiúsculas na horizontal comparam os tratamentos e letras minúsculas na vertical comparam as espécies, para cada tratamento e parte vegetal; C.V - coeficiente de variação. *T1 - Substrato; **T2 - Substrato + 4\% EA + $1 \% \mathrm{CL} ; * * \mathrm{~T} 3$ - Substrato $+6 \%$ EA $+1 \%$ CL. EA, escória de aciaria; CL, carepa de laminação 
ser afetado pelo excesso de zinco, pois interfere em múltiplas vias, causando redução na fotossíntese, inibindo o desenvolvimento das plantas, alterando as estruturas e organização celular, além de alterar a atividade das enzimas que participam do metabolismo das plantas (GUO et al., 2007).

$\mathrm{O}$ acúmulo de $\mathrm{Zn}$ pelas espécies de eucalipto (TAB. 4) ocorreu de forma diferenciada de acordo com os tratamentos. Para E. urophylla, o maior acúmulo total ocorreu no tratamento 2, i.e, $14.519 \mu \mathrm{g} \mathrm{planta}^{-1}$, enquanto que no tratamento 3 foi de $13.462 \mu \mathrm{g}$ planta $^{-1}$. A espécie E. saligna apresentou maior acumulação total de $\mathrm{Zn}$ no tratamento 3 , de $20.757 \mu \mathrm{g}$ planta $^{-1}$, enquanto que no tratamento 2 o acúmulo foi de $16.483 \mu \mathrm{g}$ planta $^{-1}$.

Ambasasespéciesdeeucaliptodemonstrarampotencial para serem utilizadas em programas de fitoestabilização, pois em projetos de remediação (PYATT, 2001), as plantas são usadas com a finalidade de absorver contaminantes biodisponíveis e retê-los nas raízes, sendo escolhidas plantas com translocação restrita às folhas, o que auxilia esse tipo de estratégia.

\section{Conclusões}

1. O tratamento do solo com os resíduos reduziu a biodisponibilidade de zinco, melhorando o desempenho das espécies estudadas;

2. A dose de $4 \%$ de escória de aciaria foi suficiente para promover o desenvolvimento das plantas, sendo que para a dose de 6\% de escória de aciaria houve maior desenvolvimento do Eucalyptus urophylla, apresentando também maior acúmulo de zinco na parte aérea, principalmente no caule;

3. A espécie Eucalyptus saligna apresentou maior acúmulo total de zinco, ficando a maior parte do zinco retida nas raízes.

\section{Referências}

ASSOCIAÇÃO BRASILEIRA DE NORMAS TÉCNICAS (ABNT). NBR 10007: Amostragem de resíduos. Rio de Janeiro, 2004. $21 \mathrm{p}$.

ACCIOLY, A. M. A. et al. Lime amelioration of zinc and cadmium toxicities for Eucalyptus camaldulensis seedlings cultivated in contaminated soil. Revista Brasileira de Ciência do Solo, v. 28, p. 775-783, 2004.

BASTA, N. T.; McGOWEN, S. L. Evaluation of chemical immobilization treatments for reducing heavy metal transport in a smelter-contaminated soil. Environmental Pollution, v. 127, n. 01, p. $73-82,2004$.
BRUNNER, I. et al. Heavy metal accumulation and phytostabilisation potential of tree fine roots in a contaminated soil. Environmental Pollution, v. 152, n.03, p. 559-568, 2008.

CARNEIRO, M. A. C.; SIQUEIRA, J. O.; MOREIRA, F. M. S. Behavior of herbaceous species in soil mixes with different degree of contamination with heavy metal. Pesquisa Agropecuária Brasileira, v. 37, n. 11, p. 1629-1638, 2002.

CASTALDI, P.; SANTONA, L., MELIS, P. Heavy metal immobilization by chemical amendments in a polluted soil and influence on white lupin growth. Chemosphere, v. 60, n. 03, p. 365-371, 2005.

CASTALDI, P.; MELIS, P. Growth and yield characteristics and heavy metals content on tomatoes grown in different growing media. Communication Soil Science Plant Analysis, v. 35, p. 85-98, 2004.

CASTALDI, P.; MULE, P.; MELIS, P. Heavy metals contents in organic matter amendments based on beached Posidonia oceanica. Annali di Chimica, v. 90, p. 741-746, 2000.

COMPANHIA DE TECNOLOGIA DE SANEAMENTO AMBIENTAL (CETESB). Relatório Estabelecimento de Valores Orientadores para Solos e Águas Subterrâneas no Estado de São Paulo. São Paulo: CETESB, 2005. p. 4.

CHEN, H. M. et al. Chemical methods and phytoremediation on soil contaminated with heavy metals. Chemosphere, v. 41, n. 01/02, p. 229-234, 2000.

CONCAS, A. et al. Metal contamination from abandoned mining sites: experimental investigation on possible remediation techniques, Land Contamination and Reclamation, v. 12, n. 01, p. 9-20, 2004.

DELL, B.; DAPING, X. Diagnosis of zinc deficiency in seedlings of a tropical eucalypti (Eucalyptus urophylla S. T. Blake). Plant Soil, v. 176, p. 329-332, 1995.

DEROME, J. Detoxification and amelioration of heavymetal contaminated forest soils by means of liming and fertilization. Environmental Pollution, v. 107, n. 01, p. 79-88, 2000.

EMPRESA BRASILEIRA DE PESQUISA AGROPECUÁRIA (EMBRAPA). Manual de métodos de análises de solos. 2. ed. Rio de Janeiro: EMBRAPA/CNPS, 1997. 212 p.

GARBISU, C. et al. Phytoremediation: A technology using green plants to remove contaminants from polluted areas. Review in Environmental Health, v. 17, n. 03, p. 75-90, 2002.

GARBISU, C; ALKORTA, I. Phytoextraction: a cost-effective plant-based technology for the removal of metals from the environment. Bioresource Technology, v. 77, n. 03, p. 229-236, 2001.

GICHNER, T. et al. Toxicity and DNA damage in tobacco and potato plants growing on soil polluted with heavy metals. Ecotoxicology and Environmental Safety, v. 65, n. 03, p. 420-426, 2006.

GRAZZIOTTI, P. H.; SIQUEIRA, J. O.; MOREIRA, F. M. S. Espécies arbóreas e ectomicorrizas em relação ao excesso de metais pesados. Tópicos em Ciência do Solo, v. 05, p. 55-105, 2003. 
GUO,T.R.; PING, Z. G.; ZHANG, Y. H. Physiological changes in barley plants under combined toxicity of aluminum, copper and cadmium. Colloid and Surfaces B: Biointerfaces, v. 57, n. 02, p. $182188,2007$.

GUPTA, A. K.; SINHA, S. Phytoextraction capacity of the plants growing on tannery sludge dumping sites, Bioresource Technology, v. 98, n. 09, p. 1788 1794, 2007.

INTERNATIONAL ORGANIZATION

FOR STANDARDIZATION (ISO). ISO 11466. Soil qualityExtraction of trace elements soluble in aqua regia. 1995.

JULIATTI, M. A. et al. Cádmio em latossolo vermelho cultivado com milho em colunas: mobilidade e biodisponibilidade. Revista Brasileira de Ciência do Solo, v. 26, n. 04, p. 1075 1081, 2002.

KABATA-PENDIAS, A.; PENDIAS, H. Trace elements in soils and plants. 2. ed. Boca Raton: CRC Press, 2001. 315 p.

KAMNEV,A. A. Phytoremediation of heavy metals: an overview. Biotechnology, v. 08, n. 06, p. 269-317, 2003.

LOMBI, E. et al. In situ fixation of metals in soilusing bauxite residue: chemical assessment. Environmental Pollution, v. 118, n. 03, p. 435-443, 2002.

MUlligAN, C. N.; YONG, R. N.; GIBBS, B. F. Remediation technologies for metal-contaminated soils and groundwater: an evaluation. Engineering Geology, v. 60, n. 01/04, p. 193-207, 2001.

PAVLÍKOVÁ, D. et al. Glutamate kinase as a potential biomarker of heavy metal stress in plants. Ecotoxicology and Environmental Safety, v. 70, n. 02, p. 223-230, 2008.

PEREIRA, A. C. C. Avaliação de estratégias de remediação de áreas contaminadas por metais pesados próximas a Baía de Sepetiba-Itaguaí. 2009. 116 f. Tese (Doutorado em Ciência do Solo), Universidade Federal Rural do Rio de Janeiro, Seropédica.

PYATT, F. B. Heavy metal accumulation in trees growing on contaminated sites in Central Europe. Ecotoxicology and Environmental Safety, v. 50, p. 60-64, 2001.

SANTOS, F. S. et al. Chemical amendment and phytostabilization of an industrial residue contaminated whth $\mathrm{Zn}$ and $\mathrm{Cd}$. Scientia Agricola, v. 64, n. 05, p. 506-512, 2007.

SOARES, C. R. F. S. et al. Fitotoxidade de cádmio para Eucalyptus maculata e E. urophylla em solução nutritiva. Revista Árvore, v. 29, p. 175-183, 2005.

TEDESCO, M. J. et al. Análise de solo, plantas e outros materiais. 2. ed. Porto Alegre, Universidade Federal do Rio Grande do Sul, 1995. 174 p. (Boletim Técnico, 5) 